\title{
Development of a CFD model for simulation of a traveling-wave thermoacoustic engine using an impedance matching boundary condition
}

\author{
Esmatullah Maiwand Sharify, Shun Takahashi, Shinya Hasegawa \\ Department of Prime Mover Engineering, Tokai University, Kanagawa 259-1292, Japan
}

\section{Corresponding Author:}

Esmatullah Maiwand Sharify, Ph.D

C +81-080-4453-1551

+81-0463-59-8293

\. esmat.maiwand@gmail.com

\begin{abstract}
Computational fluid dynamics (CFD) simulations have become prevalent tools in the numerical modeling of complex thermoacoustic phenomena. The basic problem concerning a CFD simulation of a complete system is the computational cost. To overcome this problem, a CFD simulation tool using an impedance matching boundary (IMB) condition has been developed to analyze the characterization of the flow field in a looped-tube travelling-wave thermoacoustic engine. The thermodynamic processes were simulated using a two-dimensional numerical solution for the compressible Navier-Stokes equations. By imposing an impedance matching boundary condition, flow fields around the regenerator and heat-exchanger plates were simulated. The boundary condition being defined as an acoustic load which was derived from experimental data. From the simulations, features of the flow field such as nonlinear vortex generation around the regenerator and heat-exchanger plates were observed that were not present in the analytical solutions. Furthermore, the temperature oscillations were obtained around regenerator plates, and the operating mechanism of a looped-tube travelling-wave thermoacoustic engine was characterized both qualitatively and quantitatively. The CFD tool was validated by obtaining good agreement when comparing results with those from experimental data and analytical solutions. As a result, it was concluded that CFD
\end{abstract}


simulations with this impedance matching boundary condition are effective in predicting actual flow behaviors in a travelling-wave thermoacoustic engine.

Keywords: Thermoacoustics, Traveling-wave, Impedance matching boundary condition, CFD simulation, Analytical linear theory, Compressible Navier-Stokes equation

\section{Nomenclature}

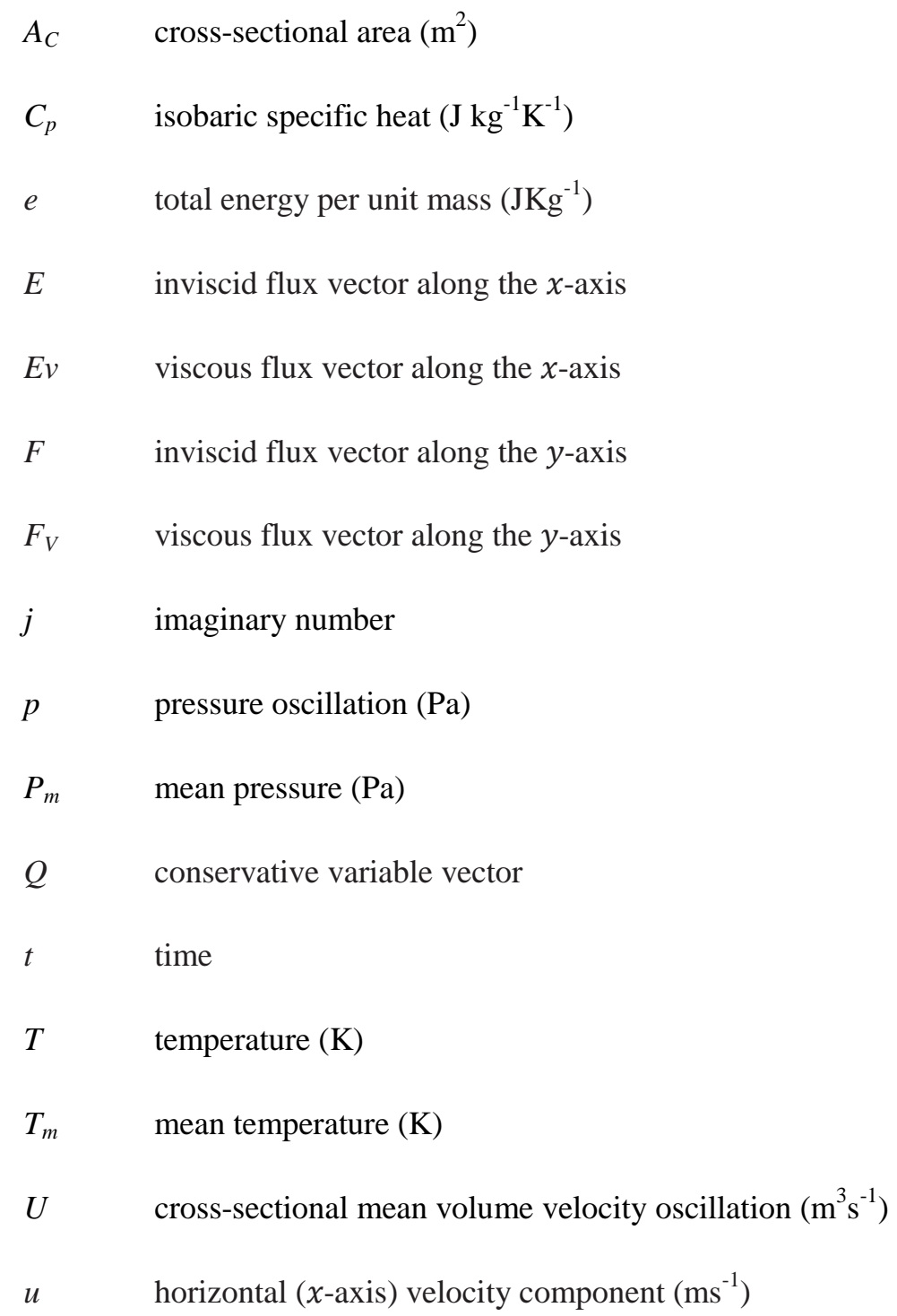


$\operatorname{vertical}\left(y\right.$-axis) velocity component $\left(\mathrm{ms}^{-1}\right)$

\section{Greek letters}

$\begin{array}{ll}\gamma & \text { ratio of specific heat } \\ \zeta, & \text { damping ratio } \\ \mu & \quad \text { dynamic viscosity }\left(\mathrm{Pa}^{*} \mathrm{~s}\right) \\ v & \text { kinematic viscosity }\left(\mathrm{m}^{2} \mathrm{~s}^{-1}\right) \\ \rho & \text { density }\left(\mathrm{kg} \mathrm{m}^{-3}\right) \\ \sigma & \text { Prandtl number } \\ \tau_{i j}: & \text { viscous stress tensor }(\mathrm{Pa}) \\ \Phi & \text { Phase difference }(\mathrm{deg} .) \\ \chi_{\omega}, & \text { thermal functions } \\ \chi_{v} & \text { viscous functions } \\ \omega & \text { angular frequency }(\mathrm{rad} \mathrm{s})\end{array}$

\section{Introduction}

The traveling-wave thermoacoustic engine, proposed by Ceperley in $1979^{[1]}$, and in 1998 Yazaki et al. ${ }^{[2]}$ built a traveling-wave thermoacoustic engine consisting of a differentially heated stack of plates in a looped tube and observed spontaneous gas oscillations of the traveling wave mode. However, over the last few decades, several studies have addressed experimental, analytical, and numerical aspects of this engine. Experimental investigations revealed the importance of understanding the underlying thermoacoustic phenomena, but the task has been complicated by a 
number of factors, which simultaneously contribute to discrepancies between theory and experiment ${ }^{[3]}$. In contrast, most of the analytical and numerical calculations performed to date were based on the linear theory of thermoacoustics. Its foundations were firmly established in a well-known series of papers by Rott and coworkers $(1969)^{[4]}$. The theory was further developed by a number of authors such as Tominaga ${ }^{[5]}$, Wheatley et al. $^{[6]}$, and Swift ${ }^{[7]}$. The linear theory of thermoacoustics makes use of several assumptions to simplify its formulation, so the operation of a thermoacoustic engine at high pressure amplitudes introduces many losses that cannot be captured by the linear theory. Subsequently, there have been many observations ${ }^{[7-9]}$ demonstrating that at high pressure amplitudes measurements deviate significantly from the predictions using linear theory. Streaming, turbulence, transition effects, and higher harmonics are mentioned as the main causes for these deviations ${ }^{[10]}$. Generally, in analytical approaches, the possibility of the formation of vortices near the stack edges are neglected ${ }^{[11]}$. To include the effects of turbulence, hysteresis, and higher harmonics systematically into the analysis remains a challenge ${ }^{[10]}$. Hence, a computational fluid dynamics (CFD) study is considered an important step towards analyzing the nonlinear phenomena.

Recently, CFD simulations have become prevalent tools in the numerical modeling of complex thermoacoustic phenomena ${ }^{[12]}$. For instance, Zink et al. ${ }^{[13]}$ used CFD to analyze the effect of resonator curvature on the thermoacoustic effect, Hariharan et al. ${ }^{[14]}$ conducted CFD simulations of an open-ended thermoacoustic prime mover with straight and tapered resonators, and Hantschk et al. ${ }^{[15]}$ employed CFD code to simulate self-excited thermoacoustic instabilities in a Rijke tube. There are also some CFD studies of traveling wave engines. Nijeholt ${ }^{[16]}$ presented a $2 \mathrm{D}$ CFD simulation study of a traveling-wave of a complete system, Scalo et al. ${ }^{[17]}$ extended Nijeholt's system to a three-dimensional model and have performed the first three-dimensional Navier-Stokes simulation of a traveling-wave thermoacoustic Stirling heat-engine, and Skariaa et al. ${ }^{[18]}$ presented the simulation studies of standing and traveling-wave thermoacoustic prime movers using software 
platforms CFD and DeltaEC ${ }^{[19]}$. Moreover, Jaworski et al. ${ }^{[20]}$ reported a CFD and experimental study of the heat transfer processes in parallel-plated heat exchanger of thermoacoustic device, and A. Toffolo et al. ${ }^{[21]}$ presented some guidelines to reduce the computational effort required to perform a CFD analysis of the thermoacoustic oscillations with commercial codes.

However, the basic problem concerning a CFD simulation of a complete system is the computational cost due to the large number of grid points employed. On the other hand, it is well known that maximum nonlinear phenomena are seen around the regenerator's unit; in contrast, nonlinear behavior in the rest of the system is relatively small. To account for the nonlinear effects, Hasegawa and Takahashi ${ }^{[22,23]}$ developed the impedance matching boundary (IMB) condition, which can realize appropriate impedance fields in the computational domain. The IMB condition was experimentally studied by Yazaki et al. ${ }^{[24]}$. K. Wang et al, ${ }^{[25]}$ applied the concept of IMB using DeltaEC for matching the acoustic impedance of the traveling wave thermoacoustic engine and the linear alternator. Hasegawa and Takahashi performed CFD simulations using the IMB condition on the parallel plates in standing-wave thermoacoustic engine where heat exchangers plates were in contact with stack plates. They defined the acoustic load and driver determined by analytical analyses studied the standing-wave thermoacoustic engine, which operates using an irreversible thermoacoustic cycle. Generally, the thermal efficiency ratio of standing-wave engines is limited. In contrast, travelling-wave thermoacoustic engines are known to reach higher thermal efficiencies than standing-wave engines, because they operate in a nearly reversible manner in terms of heat transfer within the engine core ${ }^{[11]}$. Hence, there is a need to understand the processes in oscillatory flow within the engine core of traveling-wave engines.

In this study, the CFD simulation with IMB condition is applied on thermoacoustic traveling wave engine. The IMB conditions were characterized by the acoustic impedance obtained from experimental data. The generation of nonlinear vortices and temperature oscillations around the 
detached regenerator and heat exchangers plates were our focus of interest as these cannot be evaluated by the analytical solutions. The objective was to develop a CFD simulation tool for predicting the actual behavior of the flow field around the regenerator unit in a looped-tube traveling-wave thermoacoustic engine. The developed CFD simulations with the IMB condition provided an effective approach to predict the actual behavior of flow in a traveling-wave thermoacoustic engine, and are seen as offering an important advancement in the nonlinear study of high-amplitude thermoacoustic systems. A comparison of the CFD simulation results with experimental data and analytical solutions was made to validate the model and is discussed in detail below.

\section{Experimental setup}

Here, we built a thermoacoustic engine in a looped tube and observed gas oscillations of the traveling wave mode running around the loop. A looped-tube travelling-wave thermoacoustic engine (Fig. 1) comprises an acoustic load, an ambient heat exchanger, a hot heat exchanger, a regenerator unit, two pairs of pressure sensors, a pair of thermocouples, and cylindrical pipes. The looped-tube engine was made of cylindrical PVC-U tubes and elbows with inner diameter of $40 \mathrm{~mm}$. Air at atmospheric pressure was used as a working fluid and an acoustic load was used to receive the sound waves with a set frequency of $169 \mathrm{~Hz}$. The ceramic regenerator, $30 \mathrm{~mm}$ in length with 0.6-mm-diameter cells, were sandwiched between two identical copper heat exchangers. The heat exchangers consisted of pairs of copper plates aligned in parallel with a 2.0-mm gap between each plate. Each plate was $1.0 \mathrm{~mm}$ thick and $30 \mathrm{~mm}$ in axial length. To control the temperature, an electrical heater (PD104K-30K) was wound around the hot heat exchanger, whereas chilled water (LTC-1200A) whose uncertainty was $\pm 2.0^{\circ} \mathrm{C}$, was used to keep the temperature of the ambient heat exchangers at room temperature. The hot heat exchanger supplied heat at $523 \mathrm{~K}$, whereas the ambient heat exchanger was used to divert heat to the surroundings at the ambient temperature 313 
$\mathrm{K}$, thereby creating a temperature gradient across the regenerator.

To quantify the performance of the engine, K-type thermocouples with uncertainty range of $\pm 2.5^{\circ} \mathrm{C}$, were used to monitor the temperature of the heat exchangers. The output signals from the thermocouples were recorded by a data logger (GL7000). Furthermore, two pairs of pressure transducers (PD104K-30K) were used at both sides of the regenerator unit, shown in Fig. 1. The output signals from the pressure transducers were recorded by a data logger (GL7000). Subsequently, the pressure and velocity amplitudes in the pipe, and their associated phase angle, were derived using the two-sensor method ${ }^{[26]}$. The experimental condition is shown in the Table 1 , and the models and the companies for the components are listed in Table 2.

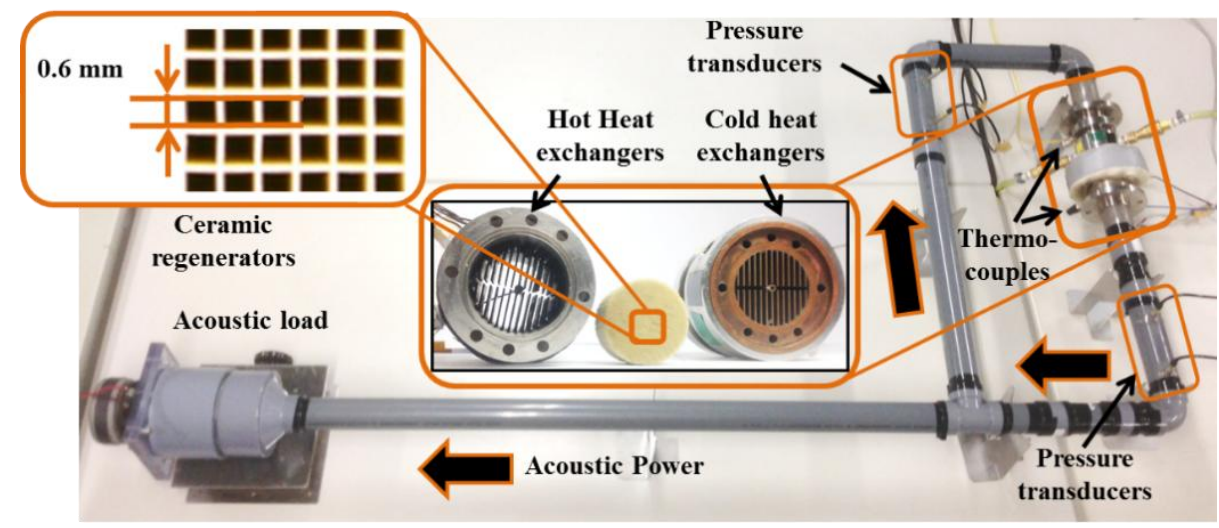

Fig. 1. Experimental setup

Table 1. The experimental condition

Frequency

Mean pressure

Working fluid

Temperature of hot heat exchanger

Temperature of cold heat exchanger
169

atmospheric

air

$523 \mathrm{~K}$

$313 \mathrm{~K}$

Table 2. The companies and the models of components

\begin{tabular}{lcl}
\hline Components & Models & Companies \\
\hline Thermocouples & T-35 (Type-K) & SAKAGUCHI E.H VOC CORP
\end{tabular}


Pressure transducers

Power supply

Low Temp Circulators

Heater

Data logger
PD104K-30K

PWR800M

LTC-1200A

2M-1-300

GL7000
JTEKT CORPORATION

KIKUSUI

ASONE CORPORATION

SAKAGUCHI E.H VOC CORP

GRAPHTEC

\section{Methods}

\subsection{Analytical method}

The analytical simulation was based on Rott's linear theory ${ }^{[4]}$ which in matrix form is

$$
\frac{d}{d x}\left[\begin{array}{l}
p \\
U
\end{array}\right]=\left[\begin{array}{c}
0 \\
\frac{-j \omega \rho_{m}}{A_{C}\left(1-\chi_{v}\right)} \\
-\frac{j \omega A_{C}}{p_{m}}\left\{1-\frac{\gamma-1}{\gamma}\left(1-\chi_{v}\right)\right\} \\
\frac{\chi_{\alpha}-\chi_{v}}{\left(1-\chi_{v}\right)(1-\sigma)} \frac{1}{T_{m}} \frac{d T_{m}}{d x}
\end{array}\right]\left[\begin{array}{c}
p \\
U
\end{array}\right]=\mathbf{A}\left[\begin{array}{c}
p \\
U
\end{array}\right]=\left[\begin{array}{ll}
A_{11} & A_{12} \\
A_{21} & A_{22}
\end{array}\right]\left[\begin{array}{c}
p \\
U
\end{array}\right]
$$

where $U$ is the cross-sectional mean volume velocity oscillation and $A_{C}$ is the cross-sectional area of the tube. Here, $p_{m}, \gamma, \sigma, T_{m}$, and $v$ are the mean pressure, ratio of specific heat, Prandtl number, mean temperature of the working gas in the tube, and kinematic viscosity, respectively. Note that the temperature gradient $d T_{m} / d x$ of the regenerator is not constant. We determined $d T_{m} / d x$ in the regenerator by performing iterations under the condition that enthalpy flow was constant at the regenerator. $\chi_{\alpha}$ and $\chi_{v}$ are the thermal and viscous thermoacoustic functions. The analytical methodology used is described in detail elsewhere ${ }^{[27]}$.

\subsection{CFD method}

CFD simulations using a low dissipative scheme were employed to capture local nonlinear phenomena $^{[28]}$. The governing equations were the two-dimensional Navier-Stokes equations for compressible flows, which were solved directly without any inclusion of turbulence models;

$$
\frac{\partial Q}{\partial t}+\frac{\partial E}{\partial x}+\frac{\partial F}{\partial y}=\frac{\partial E_{v}}{\partial x}+\frac{\partial F_{v}}{\partial y}
$$




$$
Q=\left[\begin{array}{c}
\rho \\
\rho u \\
\rho v \\
\rho e
\end{array}\right], E=\left[\begin{array}{c}
\rho u \\
\rho u^{2}+p \\
\rho u v \\
(\rho e+p) u
\end{array}\right], F=\left[\begin{array}{c}
\rho u \\
\rho v u \\
\rho v^{2}+p \\
(\rho e+p) v
\end{array}\right], E_{v}=\left[\begin{array}{c}
0 \\
\tau_{x x} \\
\tau_{x y} \\
\tau_{x x} u+\tau_{x y} v+\kappa T_{x}
\end{array}\right], F_{v}=\left[\begin{array}{c}
0 \\
\tau_{y x} \\
\tau_{y y} \\
\tau_{y x} u+\tau_{y y} v+\kappa T_{y}
\end{array}\right]
$$

Here, $Q, E, F, E_{v}, F_{v}$ denote vectors of conservative variables, and the inviscid and viscous fluxes along the $x$ - and $y$-axes. The stress tensor components are:

$$
\begin{aligned}
& \tau_{x x}=\frac{2}{3} \mu\left(2 u_{x}-v_{y}\right), \\
& \tau_{y y}=\frac{2}{3} \mu\left(2 v_{y}-u_{x}\right), \\
& \tau_{y x}=\tau_{x y}=\mu\left(u_{y}-v_{x}\right) .
\end{aligned}
$$

The pressure $p$ is related to the total energy $e$ per unit mass by the equation of state,

$$
\rho e=\frac{p}{\gamma-1}+\frac{1}{2} \rho\left(u^{2}+v^{2}\right)
$$

The temperature $T$ in the energy equation was estimated using the specific heat at constant pressure $C_{p}$ based on the ideal gas law:

$$
C_{p} T=e+\frac{p}{\rho}-\frac{1}{2}\left(u^{2}+v^{2}\right)
$$

All variables were non-dimensionalized by the freestream conditions of density, sound speed, and unit length. The relationship between the temperature and the other parameters is defined by:

$$
\begin{aligned}
& \kappa=2.34 \times 10^{-2}+7.40 \times 10^{-5}(T-373.15)^{0.76} \\
& \alpha=\left(\kappa_{t}-\kappa_{s}\right) \kappa T=\left(\frac{1}{\gamma P}-\frac{1}{P}\right) \kappa T \\
& \mu=\alpha \sigma \rho
\end{aligned}
$$

Here, $\kappa, \kappa_{t}, \kappa_{s}, \alpha, P$ and $\sigma$ are thermal conductance, isothermal compressibility, thermal diffusivity, reference pressure and Prandtl number, respectively. The inviscid fluxes are estimated by the low-dissipative switching scheme ${ }^{[28]}$. The scheme consisting of second-order low-dissipative pseudo skew-symmetric scheme and third-order general MUSCL-Roe upwind scheme which can capture sound waves clearly with suppressing the numerical dissipation. 
The finite volume method was used to discretize the above equations on equally spaced Cartesian grids with a cell-centered arrangement. The wall boundaries of objects were represented by the immersed boundary method using ghost cells of the level-set method which computes the normal vector from the object surface on the basis of a gradient operation. The cells are classified into three categories: fluid cell, ghost cell, and object cell. A schematic of the cells around a boundary is shown in Figure 2.

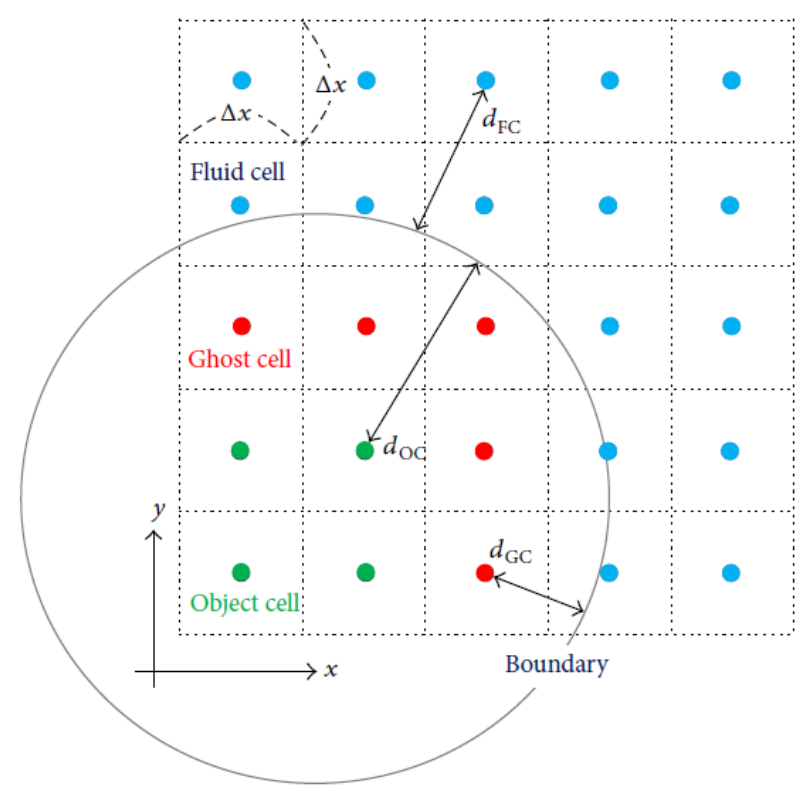

Fig 2: Cell construction and classification in the present level set method ${ }^{[28]}$.

The ghost cells behave as guard-cells between the fluids and object regions and are assigned in two layers under the present definition as follows:

$$
\left.d_{F C}\right\rangle, \quad d_{G C} \leq 0, \quad d_{G C} \geq-2 \sqrt{2} \Delta x, \quad d_{O C}\langle-2 \sqrt{2} \Delta x
$$

The no-slip boundary condition was used for the velocity and the Neumann boundary condition was imposed on the walls for the pressure. Meanwhile, isothermal boundary condition was applied for the temperature. The numerical methodology has been described in detail elsewhere ${ }^{[28]}$. 


\subsection{IMB condition}

The impedance is a concept of paramount importance in acoustics ${ }^{[29]}$ and it is one of the main parameters involved in setting boundary conditions. The IMB condition for a truncated domain of an infinite domain has been the subject of extensive research for the past decade ${ }^{[30]}$. The condition used here was developed by Hasegawa and Takahashi ${ }^{[22,23]}$ as a one-dimensional linear system which is defined as:

$$
Z=\frac{p}{u}=2 \zeta \omega_{n}+\left(\omega-\frac{\omega_{n}^{2}}{\omega}\right) j
$$

Here, $j, \zeta, \omega_{n}$, and $\omega$ are the imaginary unit, damping ratio, natural angular frequency, and angular frequency, respectively. The natural angular frequency $\omega_{n}$ was determined using experimental results. With pressure $p$ obtained from the flow simulation, the IMB condition for the acoustic load is defined as:

$$
u=\frac{p}{2 \zeta \omega_{n}+\left(\omega-\frac{\omega_{n}^{2}}{\omega}\right) j}
$$

Here, both pressure $p$ and velocity $u$ are cross-sectionally averaged values. Furthermore, the boundary condition for the sound waves imposed by the acoustic load is written

$$
u=\frac{p+f}{2 \zeta \omega_{n}+\left(\omega-\frac{\omega_{n}^{2}}{\omega}\right) j} .
$$

Here, $f$ is the external input adjusted for desirable thermoacoustic fields. The pressure $p$ in Eq. (12) was obtained from the flow simulation. The natural angular frequency of the driver $\omega_{n}$ was determined to be the same as that for the external input $f$. All values of the damping ratio $\zeta$ were set to 1.0. The implementation of the IMB condition is described in the appendix.

\subsection{Numerical condition}

In this study, a 2D CFD simulation was performed and to save computing time and expense, we restricted our computations to the flow field around the regenerator unit, using the 
IMB condition. The regenerator is configured as a stack of flat plates of equal thickness with each plate discretized using eight cells. Although a regenerator usually contains a large number of identical plates, so we further reduced computing times by configuring only four regenerator plates and assumed the structures to be periodic along the $y$ direction. Subsequently, the thermodynamics near and within the plate stack were simulated by the relevant Navier-Stokes equations for unsteady compressible flow. Air at atmospheric pressure was used as working fluid with a fixed frequency of $169 \mathrm{~Hz}$. A term linear in the temperature gradient was added to the plates as isothermal walls of $313 \mathrm{~K}$ on the left-hand side of the regenerator and $523 \mathrm{~K}$ on the right-hand side of the heat exchanger. The flow simulations were conducted over 13 cycles with constant time increments of $0.029 \mu$ s. Figure 3 shows the schematics of the flow simulation for analytical setup and computational domain.

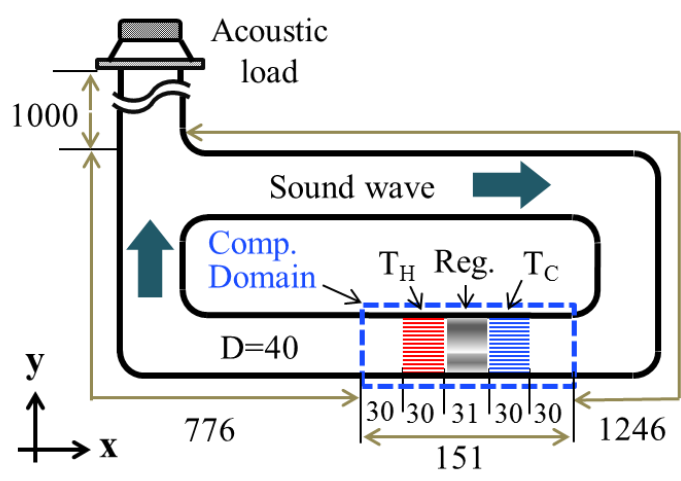

(a)

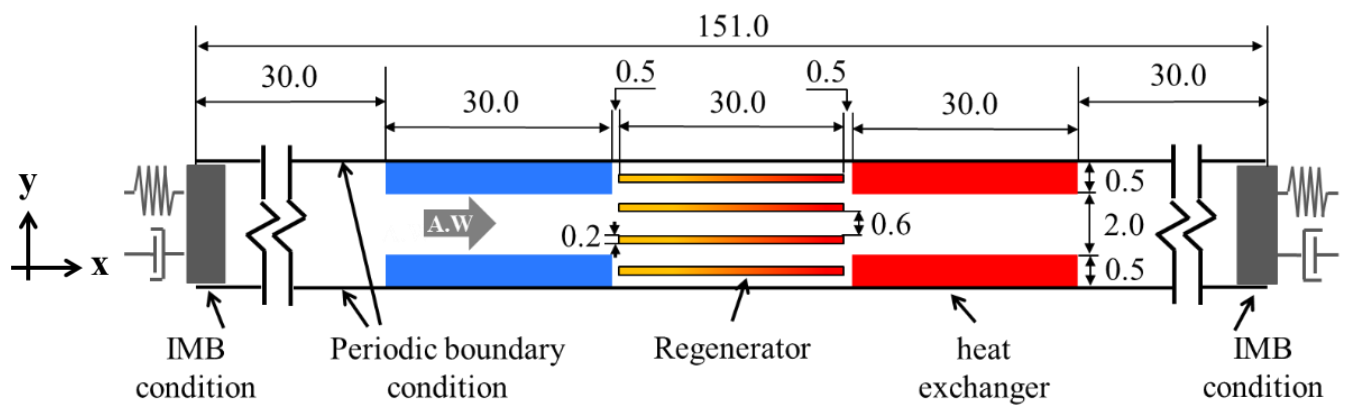

(b)

Fig. 3. Schematic of the flow simulation:

(a) analytical setup, (b) computation domain; lengths scales are in $\mathrm{mm}$. 
Table 3 summarized the specification of computational domain and boundary conditions values such pressure amplitude, velocity amplitude, phase differences for the left and the right side boundaries. The shown boundary condition values are being defined by the experimental data. Subscripts $L$ and $R$ signify the left- and right-hand side boundaries.

Table 3. Numerical conditions for the simulations.

\begin{tabular}{lccc}
\hline \multicolumn{1}{c}{ Type } & Unit & Value \\
\hline Mash size & {$[\mathrm{m}]$} & $2.5 \mathrm{E}-05$ \\
$\begin{array}{l}\text { Domain size } x \text { direction } \\
\text { Domain size } y \text { direction }\end{array}$ & {$[\mathrm{m}]$} & $1.51 \mathrm{E}-01$ \\
$\begin{array}{l}\text { Pressure on left-side boundary of the domain } P_{L} \\
\text { Pressure on right-side boundary of the domain } P_{R}\end{array}$ & {$[\mathrm{~Pa}]$} & $3.00 \mathrm{E}-03$ \\
$\begin{array}{l}\text { Velocity on left-side boundary of the domain } U_{L} \\
\text { Velocity on right-side boundary of the domain } U_{R}\end{array}$ & {$[\mathrm{~Pa}]$} & $15.17 \mathrm{E} 02$ \\
$\begin{array}{l}\text { Phase difference between } \\
\text { boundary of the domain } \varphi_{L}\end{array}$ & {$[\mathrm{~m} / \mathrm{s}]$} & $1.51 \mathrm{E} 02$ \\
Phase difference between & $1.11 \mathrm{E} 0$ \\
boundary of the domain $\varphi_{R}$ & {$[\mathrm{deg}]$.} & $0.00 \mathrm{E} 0$ \\
\hline
\end{tabular}

\subsection{Grid independence study}

To achieve grid size-independence solution, a grid independence study was done and the simulations were performed on the grids of different sizes (coarse, medium, and fine). The regenerator plates are the core structures in the computational domain, and enough mesh nodes for boundary layer along the plate thickness are the most dominant. Therefore, the regenerator plate thickness for the coarse, medium, and fine grids were discretized with the 7,8 , and 9 cells, respectively. The numerical errors associated with these grids were checked with normalized acoustic power and acoustic power gain as shown in table 4 and Fig. 4. 
Table 4. Acoustic power gain for coarse, medium and fine computational grids

\begin{tabular}{lllll}
\hline Grid type & Grid size & $\begin{array}{l}\text { Min. mesh } \\
\text { size }(\mathrm{m})\end{array}$ & $\begin{array}{l}\text { Cells along the } \\
\text { plate thickness }\end{array}$ & $\begin{array}{l}\text { Acoustic } \\
\text { power gain }\end{array}$ \\
\hline Coarse & $5491 \times 107$ & $2.75 \mathrm{E}-05$ & 7.0 & 1.241 \\
Medium & $6039 \times 119$ & $2.50 \mathrm{E}-05$ & 8.0 & 1.235 \\
Fine & $6709 \times 131$ & $2.25 \mathrm{E}-05$ & 9.0 & 1.230 \\
\hline
\end{tabular}

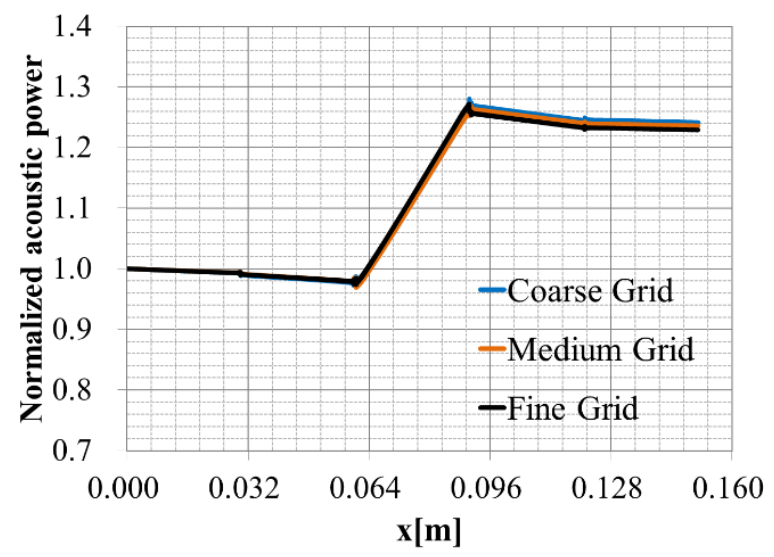

Fig. 4. Comparison of normalized acoustic power for coarse, medium, and fine computational grids

It was found that the differences in solutions for the normalized acoustic power and acoustic power gain for the different sizes of grids (coarse, medium, and fine) were around $2 \%$ and $0.4 \%$, respectively. Consequently, it was confirmed that the results are independent of the grid sizes and the results presented later are based on the medium computational grids.

\section{Results}

The major part of discussion in this section concerns the CFD results. The acoustic results such as pressure and velocity amplitudes, and the phase difference between these amplitudes, and local acoustic power were the focus of the research. Figure 5 shows ten phases for the right-hand side boundary of the computational domain. These phases provide a visual representation of the unsteady flows. The pressure and velocity values shown in the figure were averaged by the cross-sectional 
area.

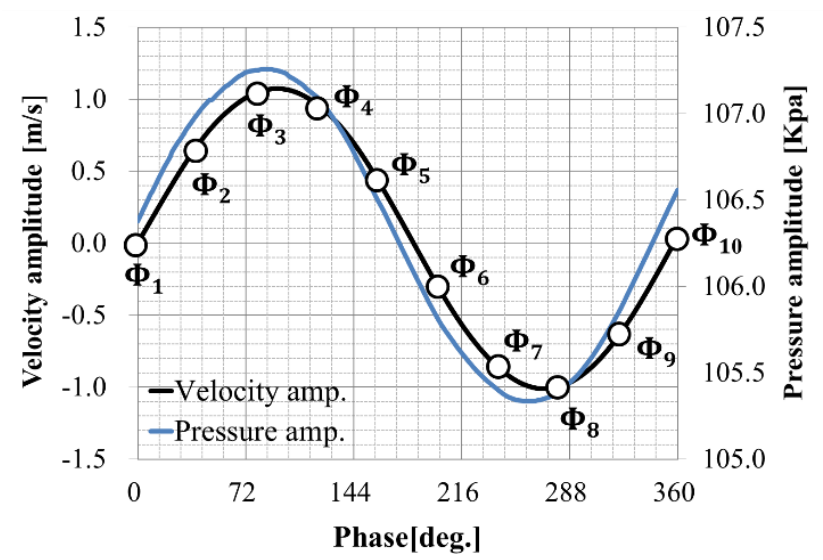

Fig. 5. Phases based on the oscillating velocity on the right-hand side boundary of the computational domain

To provide a visual rendering of the flow, the vorticity fields around the regenerator and heat exchanger plates at various phases in the acoustic cycle are presented in Figs. 6 and 7, respectively.

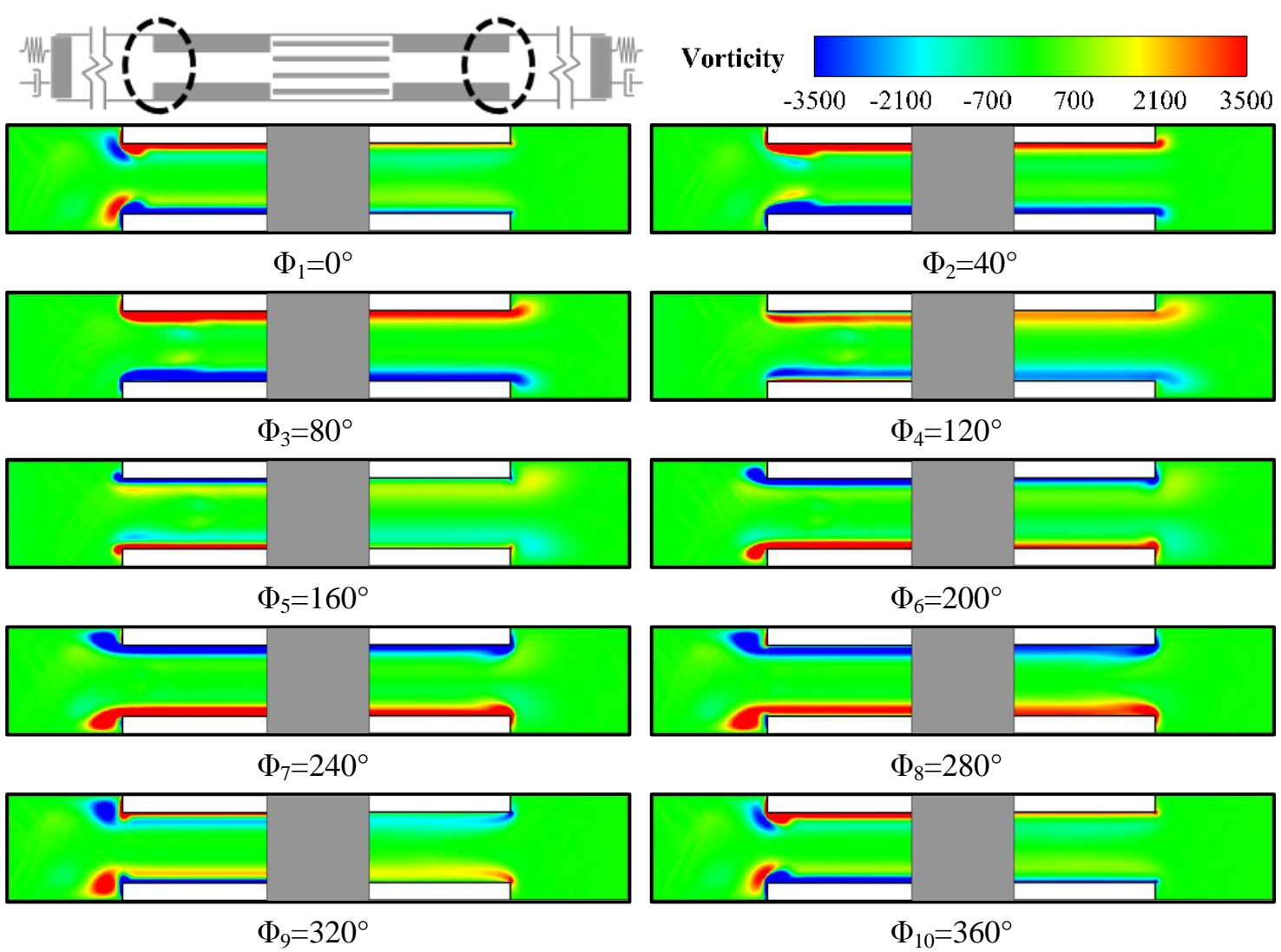

Fig. 6. Vorticity fields around heat exchangers 


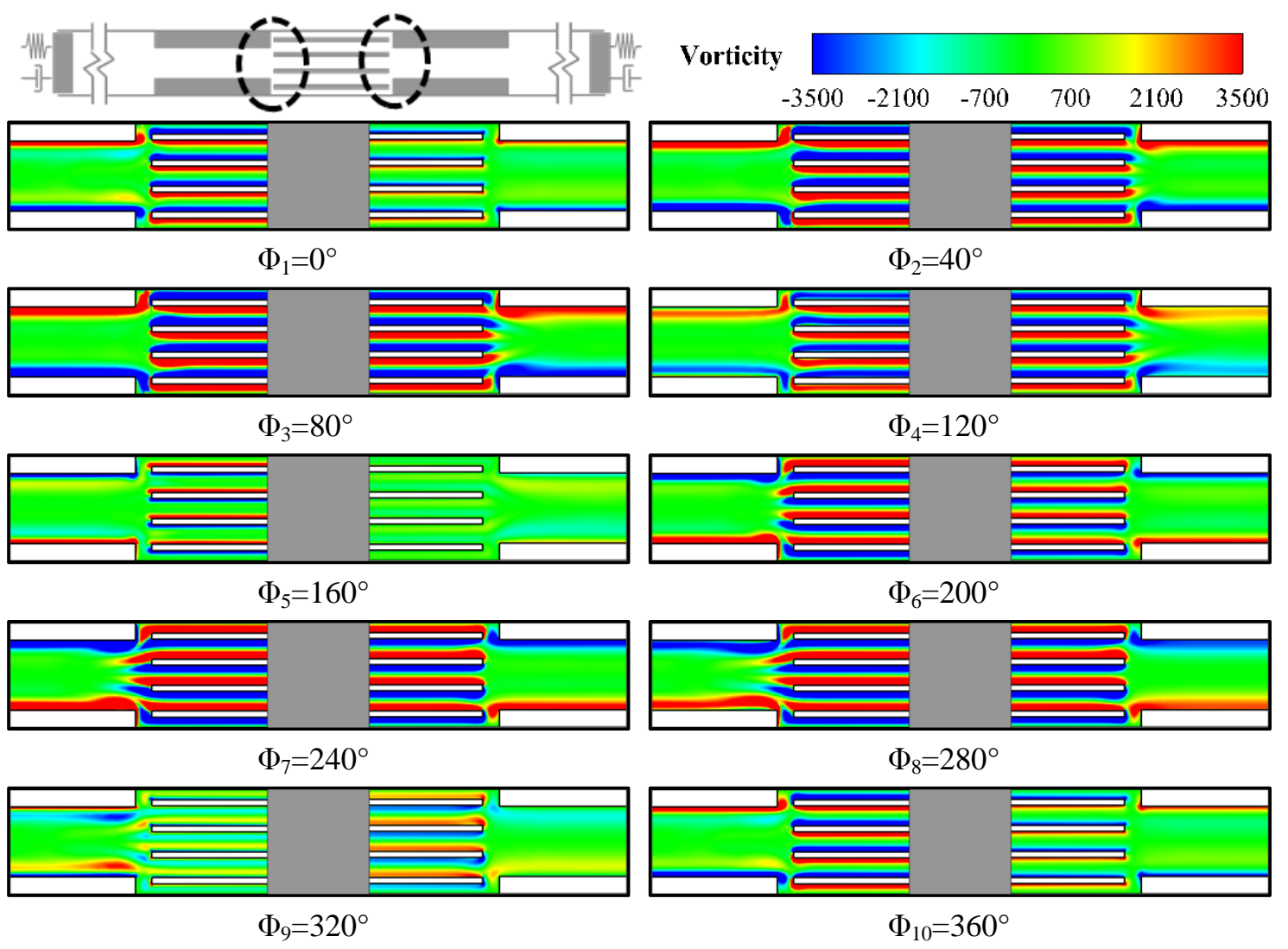

Fig. 7. Vorticity fields around regenerator plates

Vortices appear in the region near the left and right edges of the heat exchangers and regenerator plates, as the fluid flows out during the cycle. The vortices are symmetrically displaced and remain near the plates. The strongest vortices were those around the heat exchangers. Moreover, the vortices on the hot side of the heat exchanger were weaker than ones on the cold side. The weakness of these vortices is the result of a decrease in the effective Reynolds number because of high temperature and viscosity. For a clearer view of the vorticity structure, close-ups of the vorticity field around the regenerator and heat exchangers' plates are presented (Fig. 8). 


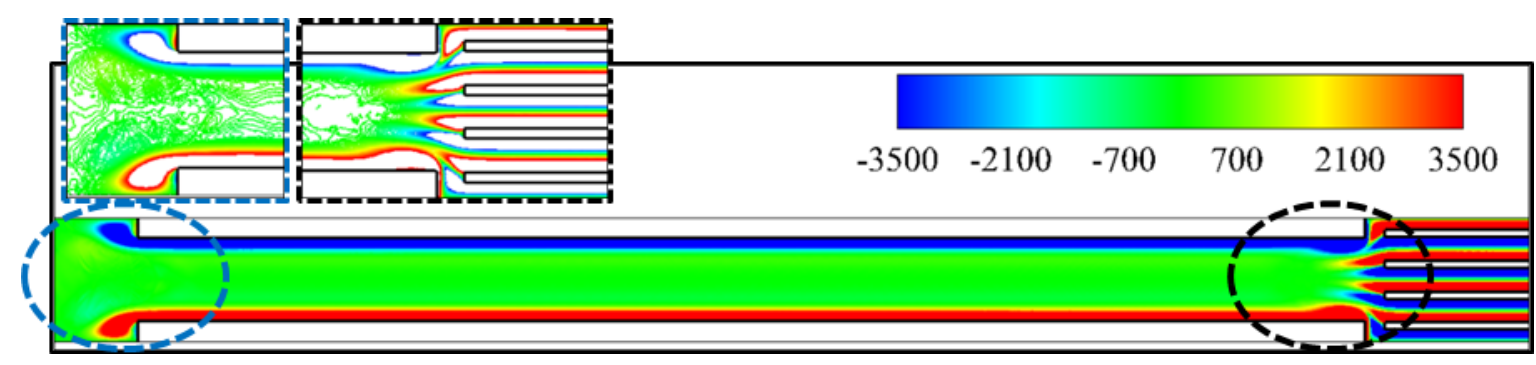

$$
\Phi_{7}=240^{\circ}
$$

Fig. 8. Vorticity field around the regenerator and heat exchangers

As it is well-known that the temperature fluctuation affects the heat transfer process and the engine performance, so the computed temperature oscillations in the regenerator and heat exchangers region are presented. To illustrate a visual thermal oscillation, the temperature contours around the regenerator and heat exchanger plates at various phases in the acoustic cycle are shown in Fig 9.

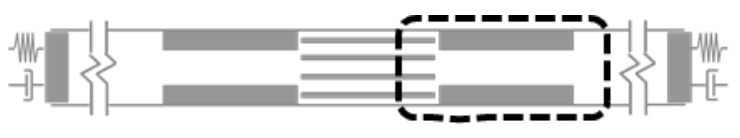

Temperature
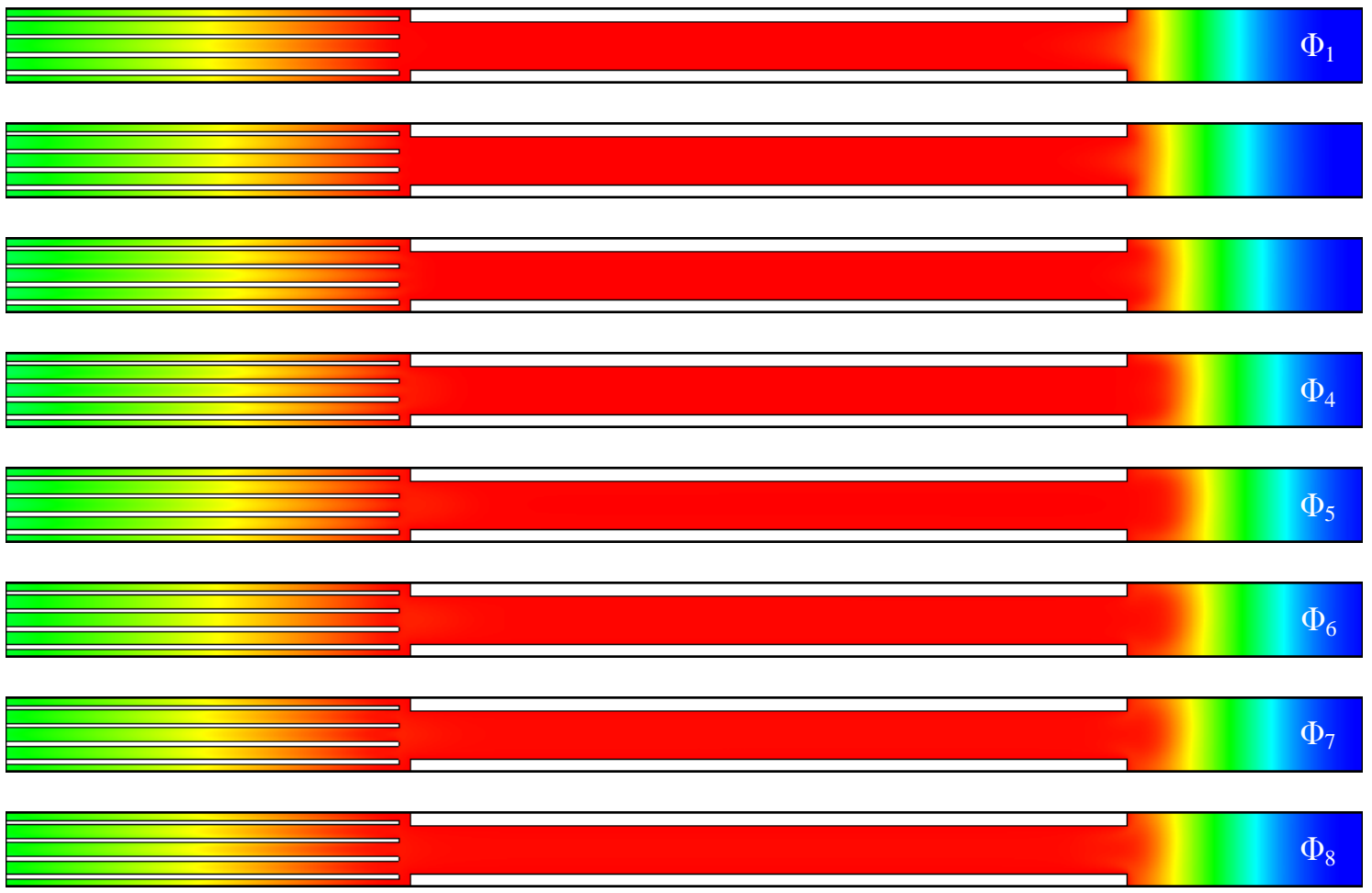


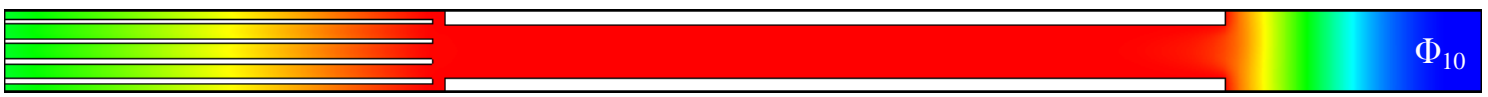

Fig. 9. Temperature fields around regenerator and heat exchanger plates

Figure 10 represents the radial - average - temperatures at three positions along the hot heat exchanger for various phases in the acoustic cycle. The blue, the black and the orange points represent the left, middle and right side positions of the hot heat exchangers, respectively.

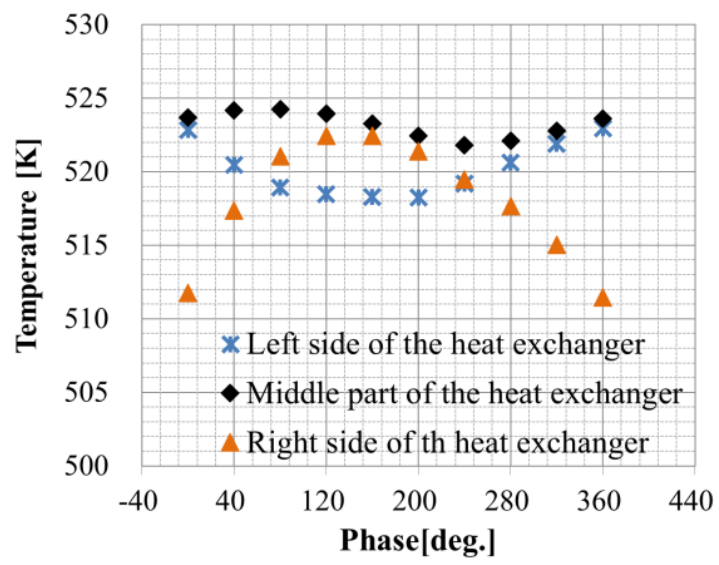

Fig. 10. Radial - average - temperatures at various phases in the acoustic cycle

From the Figs. 9-10, the temperature oscillations around regenerator and heat exchanger plates were confirmed. The temperature variation at right side of the hot heat exchanger was larger than those at the left side of the heat exchanger. In particular, the temperature between the hot heat exchanger plates at middle positon of the regenerator plates doesn't change significantly with the oscillation. Overall, the temperature of fluid in all points was lower than the temperature of the wall.

To assess the correctness of the CFD simulation, the computed results were compared with experimental data and analytical results. Axial distributions of the velocity amplitude, pressure amplitude, phase difference, and normalized acoustic power were plotted (Fig. 11). All the CFD results were calculated and recorded during the last acoustic cycle in which 763 time steps were used 
for computation.
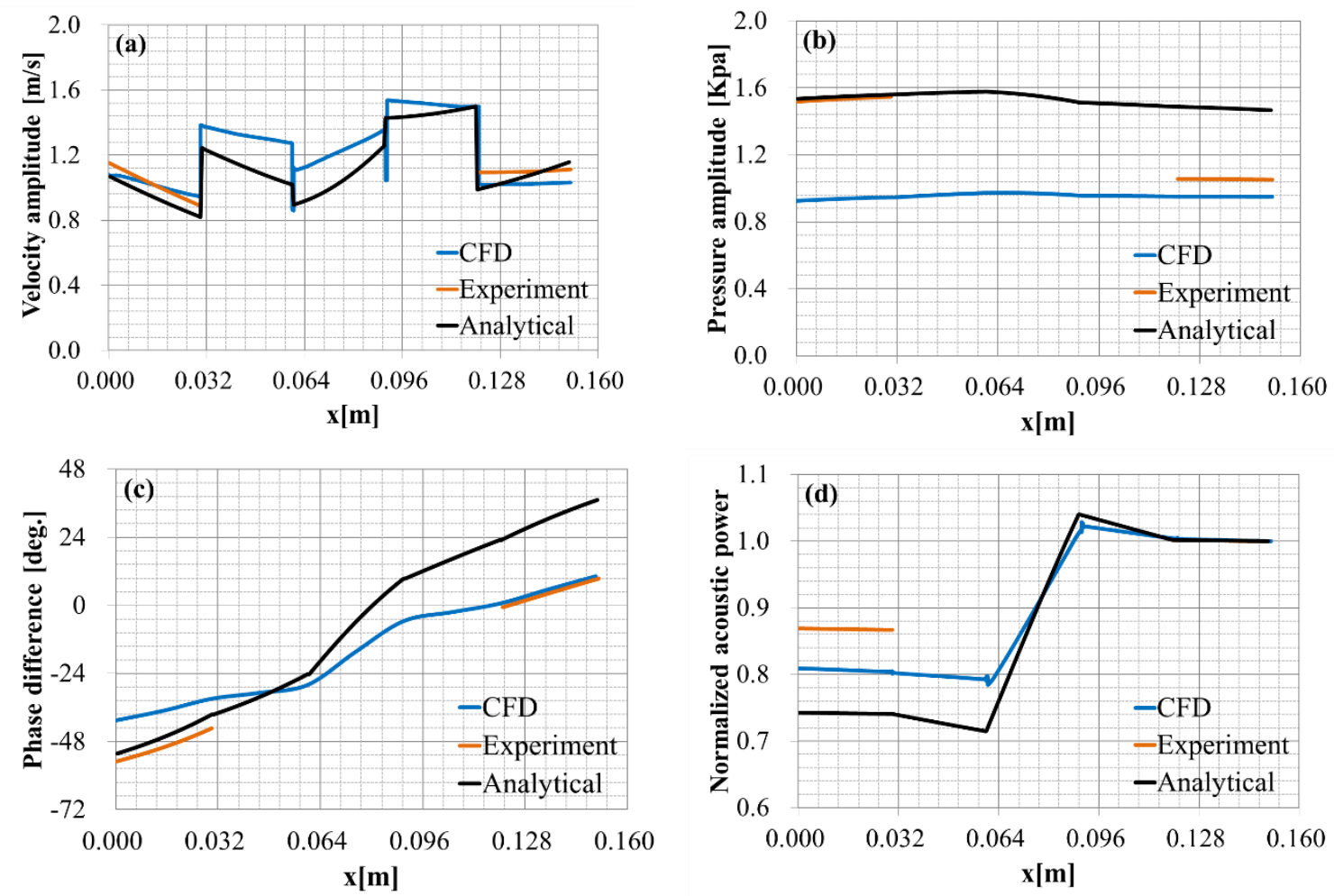

Fig. 11. Comparison of axial distributions of the acoustic parameters: (a) velocity amplitude, (b) pressure amplitude, (c) phase difference, (d) normalized acoustic power. The blue lines, the orange lines, and the black lines represent the CFD, experimental, and analytical results, respectively.

From the comparison of the velocity amplitude [Fig. 11(a)], the computed velocity amplitude exhibits good agreement with both experimental data and analytical result. In all cases, the velocity amplitudes changed because of areal variation in the heat exchanger sections. Moreover, an increase in the velocity amplitudes was observed in the regenerator section because of temperature gradients along its axial direction. Figure 11(b) presents a comparison of the pressure amplitude between the CFD, experimental data, and analytical results. Experimentally, the pressure amplitude decreases in the regenerator section. This pressure drop is attributed to thermal effects as well as three-dimensionality effects. These effects can also be seen in the distribution of the phase difference on the left-hand side of the computation domain, as evident in the relatively small phase shift with respect to the CFD results [Fig. 11(c)]. Analytically, an almost zero phase difference between 
pressure and velocity amplitudes was observed in the regenerator section.

The computed acoustic power was compared with measured values from experiment and values from analytical solutions [Fig. 11(d)]. The acoustic powers were normalized with respect to the acoustic power values on the right-hand side boundary of the domains. In all cases, a similar decrease in the acoustic powers in both cold and hot heat exchanger parts is evident. This was mainly because of viscous losses resulting from the interactions of the gas particles with the heat exchanger plates. Furthermore, the regenerator section exhibited an increase in acoustic power caused by the interaction of acoustic waves with tightly spaced plates having a temperature gradient along the axial direction. The analytic solutions showed relatively higher rise in the acoustic power with larger gradients compared with that from the CFD simulation and experimental data. In comparison with the experimental results, the CFD simulation and analytical solutions overpredicted the acoustic power gain around $7 \%$ and $17 \%$ respectively.

From the comparison of results (Fig. 11), some discrepancies were found, but they were not due to nonlinear effects round regenerator and heat exchanger plates. In both analytical and experimental cases, the entire engine was taken into account. The analytical solution was obtained from an axisymmetric calculation, and the minor losses due the tubes and elbows were not included. Therefore, the acoustic power gain for the analytical solutions showed higher value in comparison with the experimental and CFD results. For the CFD case, the boundary conditions of IMB condition were defined from the experimental data. So, the CFD results were seen to compare fairly well with those from the experimental results. However, the CFD simulations were restricted to a 2D domain and the effects of the third dimension were not included. The observed discrepancies between the CFD and experimental results are attributable to thermal and three-dimensionality effects. Moreover, in experimental setup, there was no space between regenerator and heat exchanger plates, and the heat exchanger plates blocked some flow channels of the regenerator which caused a reduction in the 
acoustic power in comparison with the analytical and the CFD results.

However, the comparison between CFD simulations, experimental data, and analytical solutions revealed good agreement. Some deviations were found but were considered insignificant. The comparison of results demonstrated the validity and efficiency of our CFD tool for characterizing the flow around the regenerator and heat exchangers plates in a looped-tube traveling-wave thermoacoustic engine. Moreover, using CFD simulation, nonlinear vortex formations at the ends of the heat exchangers and regenerator plates were observed which are not apparent in the analytical solutions. Nevertheless, in this study, a low-amplitude pressure was presumed, and hence the energy loss obtained in the CFD simulation was minor and insignificant. As a result, we conclude that CFD simulations with IMB condition characterize well both qualitatively and quantitatively the operating mechanism of the looped-tube travelling-wave thermoacoustic engine.

\section{Conclusions}

A nonlinear simulation tool was developed to analyze the characterization of the flow field around the regenerator and heat exchangers in a looped-tube travelling-wave thermoacoustic engine. The tool was validated by comparing simulation results with those from experimental data and analytical solutions. The following general observations were obtained:

a. The computational results were found to agree with the experimental data and analytical solutions.

b. Using the CFD simulation, nonlinear vortex generation around the regenerator and heat-exchange plates were observed that were not evident from the analytical solutions.

c. The CFD simulations with the IMB condition characterized both qualitatively and quantitatively the operating mechanism of a looped-tube travelling-wave thermoacoustic engine.

d. The temperature oscillations around regenerator and heat exchanger plates were confirmed. 
e. In comparison with the experimental results, the CFD simulation and analytical solutions overpredicted the acoustic power gain around $7 \%$ and $17 \%$ respectively.

f. The CFD simulations with the IMB condition provide an effective approach to predict the actual behavior of flow in a traveling-wave thermoacoustic engine, and are seen as offering an important advancement in the nonlinear study of high-amplitude thermoacoustic systems.

\section{Appendix}

The IMB condition for the acoustic load was defined using a linear system consisting of a mass, spring, and damper components with a periodic input force $p^{\prime}=P \sin (\omega t+\varphi)$ and a periodic output velocity $u^{\prime}=U \sin (\omega t)$, determined by:

$$
\begin{aligned}
& m \frac{d u^{\prime}}{d t}+c u^{\prime}+k \int u^{\prime} d t=p^{\prime} \\
& \left\{\begin{array}{l}
m \omega-\frac{k}{\omega}=\frac{P}{U} \sin \Phi \\
c=\frac{P}{U} \cos \Phi
\end{array}\right.
\end{aligned}
$$

Here, $m, c$, and $k$ are the mass, damping constant, and spring constant of the system, respectively, the

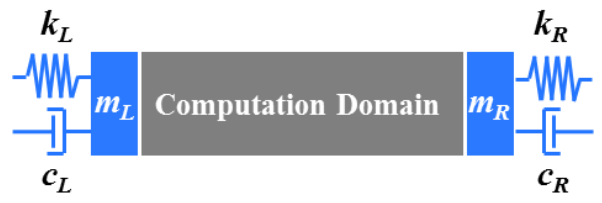

Fig. 12. Schematic of the impedance-matching boundary conditions

determination of which for each system follows from:

$$
\begin{aligned}
& \left\{\begin{array}{l}
m_{L} \omega-\frac{k_{L}}{\omega}=0 \\
c_{L}=\frac{P_{L}}{U_{L}}
\end{array}\right. \\
& \left\{\begin{array}{l}
m_{R} \omega-\frac{k_{R}}{\omega}=\frac{P_{R}}{U_{R}} \sin \Phi_{R} \\
c_{R}=\frac{P_{R}}{U_{R}} \cos 2 \Phi_{R}
\end{array}\right.
\end{aligned}
$$


In this system the left-hand side boundary with zero phase difference operated as a uniform damping system to reflect the pressure wave from the right-hand load system without any change in the frequency. Here, the parameters of $m$ and $k$ were defined based on the damping ratio $\zeta=0.5 c(m k)^{-0.5}=1.0$ The systems with parameters driven from experimental results were solved with equations as follows:

$$
\begin{aligned}
& \left\{\begin{array}{l}
m_{L} \frac{d u_{l}}{d t}+c_{L} u_{l}+k_{L} \int u_{l} d t=P_{l}^{\prime} \\
P_{l}^{\prime}=P_{r e f}-P_{l b}-\beta P_{L} \sin \left(\omega t+\Phi_{L}\right)
\end{array}\right. \\
& \left\{\begin{array}{l}
m_{R} \frac{d u_{r}}{d t}+c_{R} u_{r}+k_{R} \int u_{r} d t=P_{r}^{\prime} \\
P_{r}^{\prime}=P_{r b}-P_{r e f}
\end{array}\right.
\end{aligned}
$$

Here, $P_{\mathrm{ref}}, P_{\mathrm{lb}}$ and $P_{\mathrm{rb}}$ are reference pressure in the computational domain, cross-sectional averaged pressure at left and right boundaries, respectively. The external force expressed as $\beta P_{L} \sin \left(\omega_{t}+\Phi_{L}\right)$ was added to the left driver boundary. The coefficient $\beta$ had to be adjusted to realize desirable acoustic field because both systems on the boundaries perform damping oscillations. The obtained velocities $u_{l}$ and $u_{r}$ were imposed as the boundary values of the $x$-axis velocity components for the flow simulation. The velocity component along with $y$-axis was kept as zero in this study. The both systems were solved by fourth-order Runge-Kutta method.

\section{Acknowledgments}

This study was partially supported by the ALCA Project of the Japan Science and Technology Agency. The flow simulations in this study were conducted using the NEC SX-9 at the Cyberscience Center of Tohoku University. 


\section{References}

[1] P. H. Ceperley, A pistonless stirling engine the traveling wave heat engine, Journal of the Acoustical Society of America 66 (1979) 1508-1513.

[2] T. Yazaki, A. Iwata, T. Maekawa, A. Tominaga, Traveling wave thermoacoustic engine in a looped tube, Physical Review Letters 81 (1998) 3128-3131.

[3] G. Petculescu, L. A. Wilena, High-amplitude thermoacoustic effects in a single pore, Journal of the Acoustical Society of America 109(3) (2001) 942-948.

[4] N. Rott, Damped and thermally driven acoustic oscillations in wide and narrow tubes, Journal of Applied Mathematics and Physics 20 (1969) 230-243.

[5] A. Tominaga, Thermodynamic aspects of thermoacoustic theory, Cryogenics 35(7) (1995) $427-440$.

[6] J. C. Wheatley, T. Hofler, G. W. Swift, A. Migliori, An intrinsically irreversible thermoacoustic engine, Journal of the Acoustical Society of America 74 (1983) 153-170.

[7] G. W. Swift, Analysis and performance of a large thermoacoustic engine, Journal of the Acoustical Society of America 92 (1992) 1551-1563.

[8] M.E. Poese, Performance measurements on a thermoacoustic refrigerator driven at high amplitudes, Master's thesis, The Pennsylvania State University, United States, 1998.

[9] S. Backhaus, G.W. Swift, A thermoacoustic stirling heat engine: Detailed study, Journal of the Acoustical Society of America 107 (2000) 3148-3166.

[10] M.M. Emam, Experimental investigations on a standing-wave thermoacoustic engine, Master's thesis, Cairo University, Egypt, 2013.

[11] X. Mao, A study of fluid flow phenomena around parallel-plate stacks in a standing wave thermoacoustic device, PhD thesis, The University of Manchester, England, 2011.

[12] B. Asgharian, K. I. Mateev, Numerical modeling of thermoacoustic no-stack heat pumps and 
prime movers, Engineering Applications of Computational Fluid Mechanics 6(3) (2012) $346-355$.

[13] F. Zink, J. Vipperman, L. Schaefer, CFD simulation of thermoacoustic engine with coiled resonator, International Journal of Heat and Mass Transfer 37(3) (2010) 226-229.

[14] N.M Hariharan, P. Sivashanmugam, S. Kasthurirengan, Influence of stack geometry and resonator length on the performance of thermoacoustic engine, Applied Acoustics 73 (2012) $1052-1058$.

[15] C.C. Hantschk, D. Vortmeyer, Numerical simulation of self-excited thermoacoustic instabilities in a Rijke tube, Journal of Sound and Vibration, 277(3) (1999) 511-522.

[16] J. L. Nijeholt, M. E. H. Tijani, S. Spoelstra, Simulation of a traveling-wave thermoacoustic engine using computational fluid dynamics, Journal of the Acoustical Society of America 118(4) (2005) 2265-2270.

[17] C. Scalo, S. K. Lele, L. Hesselink, Numerical investigation of a traveling-wave thermoacoustic stirling heat engine, Center for Turbulence Research Annual Research Briefs, 2013.pp. 165-178.

[18] M. Skariaa, K. K. A. Rasheed, K. A. Shafi, S. Kasthurirengan, U. Behera, Simulation studies on the standing and traveling wave thermoacoustic prime movers, AIP Conference Proceedings 1573 (2014) 760-767.

[19] B. Ward, J. Clark, G.W. Swift, Design environment for low-amplitude thermoacoustic energy conversion, Version 6.2. Users Guide, 2008.

[20] A. Jaworski, A. Piccolo, Heat transfer processes in parallel-plate heat exchangers of thermoacoustic devices - numerical and experimental approaches, Applied Thermal Engineering 42 (2012) 145-153.

[21] A. Toffolo, M. Masi, A. Lazzaretto, Low computational cost CFD analysis of thermoacoustic oscillations, Applied Thermal Engineering 30 (2010) 544-552. 
[22] S. Takahashi, S. Hasegawa, T. Nonomura, Numerical simulation of a thermoacoustic engine by compressible Navier-Stokes equations, 46th Fluid Dynamics Conference / 32nd Aerospace Numerical Simulation Symposium, Japan, (2014).

[23] M. Senga, S. Hasegawa, Design and experimental verification of a cascade traveling-wave thermoacoustic amplifier, Journal of Applied Physics 119 (2016) 204906.

[24] H. Hatori, T. Biwa, T. Yazaki, How to build a loaded thermoacoustic engine, Journal of Applied Physics 111 (2012) 074905.

[25] K. Wang, J. Zhang, N. Zhang, D. Sun, K. Luo, J. Zou, L. Qiu , Acoustic matching of a traveling-wave thermoacoustic electric generator, Applied Thermal Engineering 102 (2016) $272-282$.

[26] A. M. Fusco, W.C. Ward, G, W. Swift, Two-sensor power measurements in lossy ducts, Journal of the Acoustical Society of America 91 (1992) 2229-2235.

[27] S. Hasegawa, T. Yamaguchi, Y. Oshinoya, A thermoacoustic refrigerator driven by a low temperature differential, high-efficiency multistage thermoacoustic engine, Applied Thermal Engineering 58 (2013)394-399.

[28] S. Takahashi, T. Nonomura, K. Fukuda, A Numerical scheme based on an immersed boundary method for compressible turbulent flows with shocks, Journal of Applied Mathematics 2014(2014), Article ID 252478, pp. 1-21.

[29] D. Noreland, Technical Report, Impedance boundary conditions for acoustic waves in a duct with a step discontinuity, Uppsala University, Sweden, 2003, pp. 1-35.

[30] S. V. Tsynkov, Numerical solution of problems on unbounded domains, Applied Numerical Mathematics 27 (1998) 465-532. 


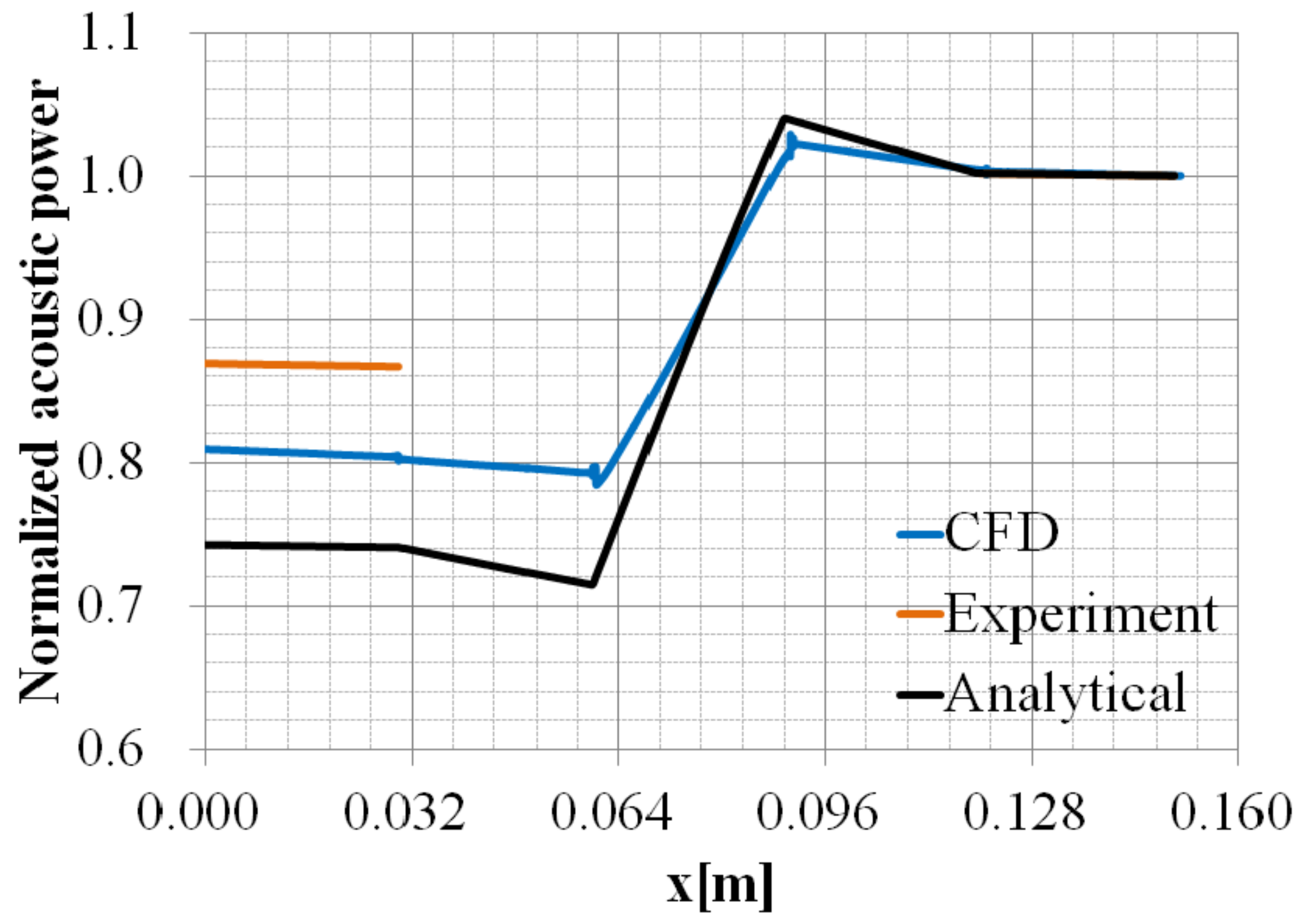

